\title{
Mathematical model of a fluxgate magnetometer
}

\author{
Pavel F. Baranov*, Vitalia E. Baranova, and Tamara G. Nesterenko \\ National Research Tomsk Polytechnic University, 634050 Tomsk, Russia
}

\begin{abstract}
In paper analytical equations for calculate the electromotive force in the measuring coil of the fluxgate magnetometer independent of the drive signal frequency content are presented. Also, the equations for estimation of the fluxgate sensitivity at any harmonic and for study fluxgates operation with a glance to the waveform and the polynomial approximation of the mean magnetization curve of the core are provided.
\end{abstract}

\section{Introduction}

The principle of a fluxgate operation is based on the change in the ferromagnet state due to the impact of two magnetic fields with different frequencies [1-3]. A simplest fluxgate configuration represents a ferromagnetic rod which allocates an $\mathrm{AC}$ driving coil and a measuring coil [4]. Due to the variable magnetic field created by the driving coil current, a magnetic core goes through the magnetic reversal along a symmetrical curve.

A change in the magnetic flux caused by the magnetic core reversal along a symmetrical curve, induces the electromotive force in a measuring coil which varies in accordance with the simple harmonic motion. Under the effect of the constant or slightly varying magnetic field affecting the magnetic core, the magnetic reversal curve changes its size and shape and becomes asymmetrical. This, in turn, modifies the value and harmonic composition of the electromotive force in the measuring coil. In particular, there appear even electromotive force harmonics the amount of which is proportional to the magnetic intensity. These even harmonics are not observed on a symmetrical curve of the magnetic reversal.

The magnetic intensity is a vector variable, and core magnetization depends not only on its orientation but also a correlation between its longitudinal and transverse sizes. Therefore, the proposed fluxgate is characterized by a direction diagram and thus can be used for measurement of the vector angles and components of the flux density.

In order to create a high-sensitive fluxgate magnetometer [5], it is advisable to analytically describe its operation and create a mathematical model.

\section{Operating principle of a single-rod fluxgate}

A simplest single-rod fluxgate, the schematic representation of which is given in figure 1, measures a constant magnetic field.

\footnotetext{
* Corresponding author: bpf@,tpu.ru
} 


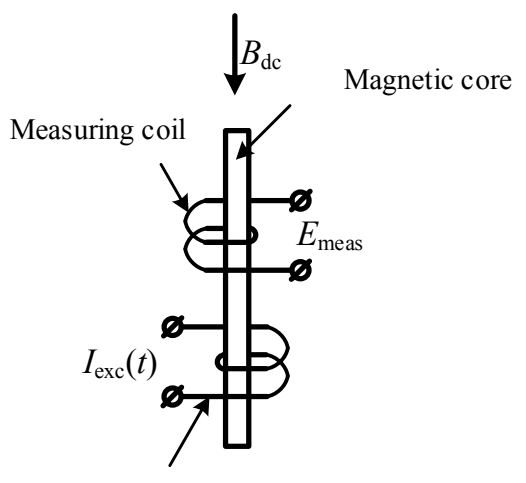

Driving coil

Fig. 1. Schematic of single-rod fluxgate.

Under the alternating exciting field $H_{\text {exc }}(t)$ which envelopes the magnetic core and the constant field $H_{d c}$ directed along the magnetic core, the magnetic intensity superposition of these fields occurs in the core bulk:

$$
H_{\Sigma}(t)=H_{\mathrm{dc}}+H_{\mathrm{exc}}(t)
$$

The flux density in the core can be then obtained from

$$
B(t)=f\left(H_{\Sigma}(t)\right)=f\left(H_{\mathrm{dc}}+H_{\mathrm{exc}}(t)\right) .
$$

The equation for the electromotive force in the measuring coil is as follows:

$$
E_{\mathrm{mes}}(t)=-s w_{2} \frac{d}{d t} B(t)
$$

where $s$ is the area of the core transverse section, $\mathrm{mm}^{2} ; w_{2}$ is the number of turns in the measuring coil.

The flux density $B(H)$ of the uniformly magnetized magnetic core in the first approximation (without regard for anisotropic and hysteresis phenomena) is usually calculated using the core magnetization function.

When the core undergoes a periodic magnetic reversal, a good agreement between theoretical calculations and experimental data is achieved for the fluxgate operation. In this case we use the mean magnetization curve which is computed by the following equation:

$$
B(H)=(B \uparrow(H)+B \downarrow(H)) / 2 .
$$

Arrows in this equation denote the flux density at ascending and descending branches of hysteresis loops. The use of the mean magnetization curve rather than the normal, is explained by the fact that the optimum operating mode for the fluxgate is the core magnetic reversal along the hysteresis loop whose ascending and descending branches slightly differ from that of the mean magnetization curve. The analysis of the fluxgate operation utilizes the polynomial approximation of the mean magnetization curve.

As the mean magnetization curve is an uneven function, it can be approximated by the uneven degree polynomial: 


$$
B=\sum_{i=1}^{j} a_{2 i-1} H^{2 i-1}
$$

where $a_{\mathrm{i}}$ is the $i$-th approximation coefficient.

For the analysis of the fluxgate operation, the dependence between the magnetic flux density $\mathrm{B}$ and intensity $\mathrm{H}$ is commonly approximated by a third-order polynomial:

$$
B=a_{1} H+a_{3} H^{3} .
$$

Using (2) and (6), we can determine the magnetic induction in the core:

$$
B(t)=a_{1} H_{\mathrm{dc}}+a_{1} H_{\mathrm{exc}}(t)+a_{3} H_{\mathrm{dc}}^{3}+3 a_{3} H_{\mathrm{dc}}^{2} H_{\mathrm{exc}}(t)+3 a_{3} H_{\mathrm{dc}} H_{\mathrm{exc}}^{2}(t)+a_{3} H_{\mathrm{exc}}^{3}(t) .
$$

When the sinusoidal alternating current excites the fluxgate, i.e. $I_{\text {exc }}(t)=I_{\text {exc } \cdot m} \sin (\omega \mathrm{t})$, the equation for the magnetic intensity can be written as

$$
H_{\text {exc }}(t)=H_{\text {exc.m }} \sin (\omega t)=\frac{w_{1}}{l} I_{\text {exc.m }} \sin (\omega t)
$$

where $I_{\text {exc } m}$ is the amplitude of excitation current, A; $w_{1}$ is the number of turns in a driving coil, $\mathrm{m} ; \omega$ is the frequency of excitation current, $\mathrm{rad} / \mathrm{s}$.

Substituting (7) into (3) in view of (8), we obtain the electromotive force in the measuring coil of the single-rod fluxgate:

$$
\begin{aligned}
& E_{\text {mes }}(t)=\left[\left(a_{1}+3 a_{3} H_{\mathrm{dc}}^{2}+\frac{3 a_{3} H_{\text {exc.m }}^{2}}{4}\right) \omega H_{\text {exc.m }} \cos (\omega t)+\right. \\
& \left.+3 a_{3} \omega H_{\mathrm{dc}} H_{\text {exc.m }}^{2} \sin (2 \omega t)+\frac{3 a_{3} \omega H_{\text {exc.m }}^{3} \cos (3 \omega t)}{4}\right] s w_{2}
\end{aligned}
$$

It follows from (9), that the useful component proportional to the measured flux density in a constant magnetic field, is on the excitation frequency $\omega$ and doubled excitation frequency $2 \omega$. At the same time, the output signal is noisy on $3 \omega$ frequency. Therefore, a complex devise based on band-pass filters or synchronous detectors is required to detect the useful component in the output signal of the single-rod fluxgate. For example, methods of synchronous detection of the fluxgate maximum sensitivity imply the use of device with four synchronous detectors. The first two detectors sense in-phase and quadrature components of the output signal on the excitation frequency $\omega$, while the second two sense them on the doubled excitation frequency $2 \omega$.

For the reasons outlined earlier, single-rod fluxgates are not applied in practice. A differential fluxgate both with open (two-rod) and closed (toroid) cores has become widespread [6-7].

\section{Differential fluxgate}

Schematic view of the differential fluxgate with open core are given in figure 2. 


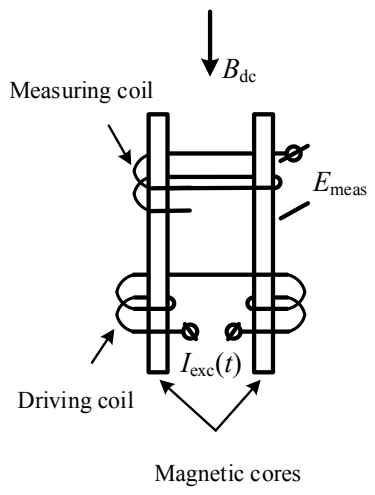

Fig.2. Differential fluxgate.

Let us consider the operation of the differential fluxgate with open core during measurement of the constant magnetic field. This fluxgate incorporates two driving coils with anti-parallel connection. The excitation current $I_{e x c}(t)$ in them creates the magnetic intensity or excitation fields $H_{e x c}(t)$ in each core. These excitation fields are characterized by the identical amplitude and phase opposition. In presence of the constant field $H_{d c}$ directed along the core as shown in figure 2, superpositions of the excitation fields in the core bulk are calculated as:

$$
\begin{aligned}
& H_{\Sigma 1}(t)=H_{\mathrm{dc}}+H_{\mathrm{exc}}(t) ; \\
& H_{\Sigma 2}(t)=H_{\mathrm{dc}}-H_{\mathrm{exc}}(t),
\end{aligned}
$$

Let us assume that the cores are identical. Their flux density is then obtained through the following equation, anisotropy and hysteresis being neglected:

$$
\begin{aligned}
& B_{1}(t)=f\left(H_{\Sigma 1}(t)\right)=f\left(H_{\mathrm{dc}}+H_{\mathrm{exc}}(t)\right) ; \\
& B_{2}(t)=f\left(H_{\Sigma 2}(t)\right)=f\left(H_{\mathrm{dc}}-H_{\mathrm{exc}}(t)\right) .
\end{aligned}
$$

The electromotive force induced in the measuring coil which, according to figure 2 , envelopes both cores, is defined as

$$
E_{\mathrm{mes}}(t)=-s w_{2}\left(\frac{d}{d t}\left(B_{1}(t)+B_{2}(t)\right)\right),
$$

Using (10) and (6), we obtain the equation, which describes the flux density:

$$
\begin{aligned}
& B_{1}(t)=a_{1} H_{\mathrm{dc}}+a_{1} H_{\mathrm{exc}}(t)+a_{3} H_{\mathrm{dc}}^{3}+3 a_{3} H_{\mathrm{dc}}^{2} H_{\mathrm{exc}}(t)+3 a_{3} H_{\mathrm{dc}} H_{\mathrm{exc}}^{2}(t)+a_{3} H_{\mathrm{exc}}^{3}(t) \\
& B_{2}(t)=a_{1} H_{\mathrm{dc}}-a_{1} H_{\mathrm{exc}}(t)+a_{3} H_{\mathrm{dc}}^{3}-3 a_{3} H_{\mathrm{dc}}^{2} H_{\mathrm{exc}}(t)+3 a_{3} H_{\mathrm{dc}} H_{\mathrm{exc}}^{2}(t)-a_{3} H_{\mathrm{exc}}^{3}(t) .
\end{aligned}
$$

In the case of the fluxgate excitation by the sinusoidal alternating current, the substitution of (13) in (12) in view of (8) allows us to derive the electromotive force in the measuring coil:

$$
E_{\mathrm{mes}}(t)=6 s w_{2} a_{3} \omega H_{\mathrm{dc}} H_{\mathrm{exc} . \mathrm{m}}^{2} \sin (2 \omega t) \text {. }
$$


So, unlike the single-rod fluxgate, the output signal of the differential fluxgate contains a useful component proportional to the measured flux density in a constant magnetic field just on the doubled excitation frequency $2 \omega$.

The traditional analysis of the fluxgate operation which uses a third-order polynomial as in (6), is rather simplified and qualitative similar to a grapho-analytical method. The quantitative analysis is provided by the approximation of $B / H$ dependence by higher order polynomials.

Let us analyze the differential fluxgate operation using the following approximation of $B / H$ dependence:

$$
B=a_{1} H+a_{3} H^{3}+a_{5} H^{5} .
$$

In the case of the fluxgate excitation by the sinusoidal alternating current, we insert (15) in (12). And in view of (11) and (8), the electromotive force in the measuring coil is found from

$$
E_{\text {mes }}(t)=s w_{2} \omega\left[-2 H_{\mathrm{dc}} H_{\text {exc.m }}^{2}\left(3 a_{3}+10 a_{5} H_{\mathrm{dc}}^{2}+5 a_{5} H_{\text {exc.m }}^{2}\right) \sin (2 \omega t)+5 a_{5} H_{\mathrm{dc}} H_{\text {exc.m }}^{4} \sin (4 \omega t)\right](16
$$

We can now proceed analogously to (16) when using the approximating dependence of the seventh order:

$$
B=a_{1} H+a_{3} H^{3}+a_{5} H^{5}+a_{7} H^{7},
$$

and thus obtain the electromotive force in the measuring coil:

$$
\begin{aligned}
& E_{\text {meas }}(t)=s w_{2} \omega\left[-\frac{1}{8} H_{\mathrm{dc}} H_{\text {exc.m }}^{2}\left(48 a_{3}+160 a_{5} H_{\mathrm{dc}}^{2}+\right.\right. \\
& \left.+80 a_{5} H_{\text {exc. } \mathrm{m}}^{2}+336 a_{7} H_{\mathrm{dc}}^{4}+105 a_{7} H_{\text {exc. }}^{4}+560 a_{7} H_{\mathrm{dc}}^{2} H_{\text {exc.m }}^{2}\right) \sin (2 \omega t)+ \\
& \left.+\frac{1}{2} H_{\mathrm{dc}} H_{\text {exc.m }}^{4}\left(10 a_{5}+70 a_{7} H_{\mathrm{dc}}^{2}+21 a_{7} H_{\text {exc.m }}^{2}\right) \sin (4 \omega t)+\frac{-21}{8} a_{7} H_{\mathrm{dc}} H_{\text {exc.m }}^{6} \sin (6 \omega t)\right]
\end{aligned}
$$

As can be seen from (16) and (18), the useful component proportional to the constant magnetic field measured, is observed in the output signal not only at the doubled excitation frequency $2 \omega$, but also at $4 \omega$ and $6 \omega$ frequencies.

The analysis of (16) and (18) allows us to propose the procedure for processing signals from the fluxgate sensor in order to enhance the fluxgate sensitivity due to measuring signals not only at the doubled excitation frequency but also at other even frequencies with the subsequent summing the results.

In practice, not only sinusoidal but also rectangular and triangular waveforms are used in the capacity of a drive signal of fluxgate magnetometers. So, it is advisable to derive a general equation for computing the electromotive force in the measuring coil independent of the waveform. Let us write the equation for the magnetic intensity of exciting field as a Fourier series without the constant component:

$$
H_{\mathrm{exc}}(t)=H_{\mathrm{exc} . \mathrm{m}} \sum_{g=1}^{m}\left(b_{g} \cos (g \omega t)+c_{g} \sin (g \omega t)\right)
$$

where $g$ is the number of harmonics in the drive signal; $b_{g}, c_{g}$ are Fourier serial expansion coefficients. 
Substituting (5) into (12) in view of (11) and 19), we obtain the resulting statement for the electromotive force:

$$
\begin{gathered}
E_{\text {mes }}(t)=-s w_{2} H_{\mathrm{q}}(t) \sum_{i=1}^{j} a_{2 i-1}(2 i-1)\left[\left(H_{\mathrm{dc}}+H_{\mathrm{exc}}(t)\right)^{2 i-2}-\left(H_{\mathrm{dc}}-H_{\text {exc }}(t)\right)^{2 i-2}\right] ; \\
H_{\mathrm{q}}(t)=\frac{d H_{\text {exc }}(t)}{d t}=\omega H_{\text {exc.m }} \sum_{g=1}^{m}\left(b_{g} \cos (g \omega t+\pi / 2)+c_{g} \sin (g \omega t+\pi / 2)\right) g .
\end{gathered}
$$
(20):

Now we can derive the differential fluxgate sensitivity at any harmonics according to

$$
\begin{gathered}
S_{g}=\lim _{T \rightarrow \infty} \frac{1}{E T} \int_{0}^{T} \frac{d E_{\mathrm{mes}}(t)}{d H_{\mathrm{dc}}} \sin (g \omega t) d t= \\
=\lim _{T \rightarrow \infty} \frac{1}{E T} \int_{0}^{T}-s w_{2} H_{\mathrm{q}}(t) \sum_{i=1}^{j} a_{2 i-1}(2 i-1)(2 i-2)\left[\left(H_{\mathrm{dc}}+H_{\mathrm{exc}}(t)\right)^{2 i-3}-\right. \\
\left.-\left(H_{\mathrm{dc}}-H_{\mathrm{exc}}(t)\right)^{2 i-3}\right] \sin (g \omega t) d t .
\end{gathered}
$$

This equation facilitates the analytical computation of the differential fluxgate sensitivity independent of the frequency content of the drive signal at a given approximation degree for $B=f(H)$ function.

\section{Conclusions}

The obtained analytical equations allowed us to calculate the electromotive force in the measuring coil of the fluxgate magnetometer independent of the drive signal frequency content. Also, the obtained equations provided the estimation of the fluxgate sensitivity at any harmonic and were used to study its operation with a glance to the waveform and the polynomial approximation of the mean magnetization curve of the core.

This research was conducted in Tomsk Polytechnic University and the research of operating principle of a single-rod fluxgate financially supported by the Ministry of Education and Science of the Russian Federation (agreement No.14.578.21.0232, unique identifier RFMEFI57817X232). The research of differential fluxgate model is funded from Russian Science Foundation (RSF), Grant Number 17-7910083.

\section{References}

1. P. Ripka, IEEE Sensors journal 10, 1108 (2010)

2. S. V. Uchaikin, LT21, 2809 (1996)

3. J. E. Lenz, IEEE Sensors journal 78, 631 (1990)

4. P. Ripka, Magnetic Sensors and Magnetometers (Artech house, 2000)

5. V. E. Baranova, et al. XXI IMEKO World Congress, (2015)

6. Baschirotto, Measurement 43, 46 (2010)

7. Hava Can, Uğur Topal, J Supercond Nov Magn 28, 1093 (2015) 